\title{
Asociación entre participación parental en los estudios, clima familiar y confianza del estudiantado con la propensión de deserción escolar del Colegio de Bachilleres Militarizado "General Mariano Escobedo" del Estado de Nuevo León (CBM)
}

\author{
Lozano Treviño, David Fernando; Maldonado Maldonado, Lauro \\ Asociación entre participación parental en los estudios, clima familiar y confianza del estudiantado con la \\ propensión de deserción escolar del Colegio de Bachilleres Militarizado "General Mariano Escobedo" del Estado \\ de Nuevo León (CBM) \\ Revista Educación, vol. 45, núm. 2, 2021 \\ Universidad de Costa Rica, Costa Rica \\ Disponible en: https://www.redalyc.org/articulo.oa?id=44066178031 \\ DOI: https://doi.org/10.15517/revedu.v45i1.42846
}

\section{(c) (1) $(9)$}

Esta obra está bajo una Licencia Creative Commons Atribución-NoComercial-SinDerivar 3.0 Internacional. 


\section{Asociación entre participación parental en los estudios, clima familiar y confianza del estudiantado con la propensión de deserción escolar del Colegio de Bachilleres Militarizado "General Mariano Escobedo" del Estado de Nuevo León (CBM)}

Parental Participation, Family Atmosphere and Student Confidence: Relationship to the School Dropout Rate at the Bachilleres Militarizado "General Mariano Escobedo" School (CBM) in Nuevo León, Mexico

\author{
David Fernando Lozano Treviño \\ Universidad Autónoma de Nuevo León y Colegio de \\ Bachilleres Militarizado "General Mariano Escobedo", \\ México \\ davidflozano@gmail.com \\ iD https://orcid.org/0000-0002-5455-4223
}

\author{
Lauro Maldonado Maldonado \\ Universidad Autónoma de Nuevo León, México \\ lamaldon@hotmail.com
}

iD https://orcid.org/0000-0002-5071-0348
DOI: https://doi.org/10.15517/revedu.v45i1.42846

Redalyc: https://www.redalyc.org/articulo.oa?

$\mathrm{id}=44066178031$

\section{Resumen:}

Objetivos: Examinar los niveles de participación parental en los estudios de sus hijas e hijos, el clima familiar, la confianza del estudiantado y la propensión de deserción escolar en el alumnado del CBM y asociar la participación parental en los estudios de sus hijas e hijos, el clima familiar y la confianza del estudiantado con la propensión de deserción escolar en el CBM. Metodología :El estudio fue descriptivo y correlacional con una muestra de 350 estudiantes. Resultados: La media de la participación parental fue de 3.87; del clima familiar 4.11; de la confianza del estudiantado 3.76, y de la propensión de deserción escolar 1.69. Resaltaron las correlaciones entre clima familiar y participación parental con 0.43 ; confianza del estudiantado con participación parental y clima familiar, 0.21 y 0.36 , respectivamente, y la correlación negativa entre confianza del estudiantado con la propensión de deserción escolar de - 0.25 . El modelo solo explicó el 5.4\% del fenómeno con asociaciones de forma negativa de la confianza y el clima familiar con un peso de -0.246 y -0.014 , con la inclinación por abandonar los estudios. Conclusiones: Se cumplieron los objetivos de la investigación, los cuales indicaron que el clima familiar y la confianza de las alumnas y los alumnos del CBM favorecen la permanencia escolar, mientras que la participación de las madres y los padres en los estudios de sus hijas e hijos es recomendable. Además, se identificó que las propensiones de desertar por el alumnado del CBM son escasas. Se recomienda fomentar la participación parental en el desempeño académico de sus hijas e hijos, propiciar espacios familiares de estimulación para la lectura, reflexión y el aprendizaje, concientizar sobre la importancia del estudio e integrar al alumnado en actividades académicas, deportivas, culturales y artísticas organizadas por la institución para acrecentar el sentido de pertenencia, entre otras.

Palabras Clave: Confianza, Deserción escolar, Educación media superior, Familia, Participación.

\section{Abstract:}

Objectives: To examine the extent of parental engagement in their children's schooling associated with the family atmosphere and how that impacts student confidence and, ultimately, school dropout rates. Methodology: A descriptive and correlational study with a sample of 350 students. Results: The average values for parental engagement was 3.87, family atmosphere, 4.11, student confidence, 3.76 and school dropout propensity, 1.69. The study highlights a correlation between family environment and parental participation of 0.43 , student confidence resulting from their parent's participation in their schooling of 0.21 and 0.36 for family environment. There was a negative correlation between student confidence and school dropout propensity of -0.25 . Just the $5.4 \%$ of the phenomenon was explained by the model with negative associations between confidence and family environment with a weight of -0.246 and -0.014 with the inclination to abandon studies. Conclusions: Based on the fulfilled study objectives, CBM students' family environment and student confidence favors school attendance. More parental participation in their children's schooling is recommended, however, to deter the propensity of student dropout rates which was low at CBM, overall. It also 
serves to promote opportunities for reading as a family, reflection and raising awareness about the importance of learning. Parental participation also encourages students to engage in academic, sports as well as cultural and artistic activities offered by the school and increase their self-worth and sense of belonging.

KEYWORDS: Self-confidence, School Dropout, High School Education, Family, Participation.

\section{INTRODUCCIÓN}

La Educación Media Superior (EMS) es el nivel donde se adquieren competencias para insertarse en puestos laborales o para incorporarse a la Educación Superior ES), además, forma parte del ejercicio de la ciudadanía y de la vida social. Comúnmente se cursa entre los 15 y 17 años, más quien no lo hace, enfrenta un elevado nivel de vulnerabilidad, pues su desarrollo se merma (Landeros, 2012). La meta es que el alumnado se concientice de que la enseñanza en este nivel tiene sentido en función de lo que sigue: el empleo o la ES (Poy, 2010). También, que cuando se finaliza el bachillerato, se tiene la oportunidad de acceder a mejores puestos de trabajo, se incrementan los ingresos, se prepara para la vida, se adquiere mayor competencia para la toma de decisiones y se fortalecen los conocimientos en comunicación y matemáticas (Secretaría de Educación Pública, 2014a).

No obstante, la reprobación y la incapacidad de estimular la permanencia de las personas jóvenes son los retos más grandes que enfrenta el bachillerato (Corzo y Reyes, 2017), donde intervienen numerosos factores, entre ellos, familiares y psicológicos. Aquellas o aquellos estudiantes que fracasan tienden a paralizar su proceso de formación; desertan sin adquirir las competencias necesarias. La falla educativa se evidencia desde que no se aprueba alguna materia (Vidales, 2009), y en donde las familias del alumnado y los aspectos conductuales y procesos mentales del propio estudiantado pudieran incidir para superar dichas fallas.

Así, el presente estudio tiene como objetivos: 1) analizar los niveles de los factores participación parental en los estudios de sus hijas e hijos, clima familiar, confianza del estudiantado y propensión de deserción escolar del CBM y 2) asociar los factores participación parental en los estudios de sus hijas e hijos, clima familiar y confianza del estudiantado con la propensión de deserción escolar del CBM. Con dichos propósitos se establece la hipótesis: a mayor participación parental en los estudios, mejor clima familiar, mayor confianza del estudiantado y menor propensión a la deserción escolar del CBM.

La investigación se ha organizado con base en los antecedentes y el marco teórico que detallan los factores o constructos analizados; en seguida, se precisa el método empleado, para después discutir los resultados, concluir y proponer algunas recomendaciones, tanto para el CBM como para cualquier institución de EMS.

\section{Antecedentes}

La deserción escolar se entiende como el acto de renunciar a la preparación académica por cualquier motivo, o bien, es la proporción demográfica entre 18 y 24 años que no ha cumplido con sus estudios de bachillerato, o que tras finalizar la educación básica no continúa con ningún tipo de formación en el último mes (RuizRamírez, García-Cue y Pérez-Olvera, 2014; Consejería de Educación de la Junta de Casilla y León [CEJC], 2013). La deserción se da cuando el estudiantado suspende o se desengancha del sistema y abandona los deberes lectivos por problemas familiares, económicos, psicológicos o escolares (Velázquez y González, 2017; Silvera, 2016).

Cuando alguien de la población estudiantil deserta, se priva de oportunidades y se genera desigualdad. Es el fin de un vigoroso y aglomerado proceso de desvinculación de la escuela (Díazy Osuna, 2017; Márquez, 2016; Salvà-Mut, Oliver-Trobat y Comas-Forgas, 2014), que inicia desde el primer año de primaria, pero se detona en la adolescencia, cuando las personas jóvenes experimentan cambios físicos, psicológicos, emocionales y 
sociales difíciles, que a veces son desconcertantes, es la etapa donde hay mayor deserción escolar (Velasco y Moreno, 2016; Secretaría de Educación Pública, 2014c).

Por otra parte, comprende una pérdida para el gobierno, pues no se aprovecha el presupuesto ni impacta en la calidad de vida, además de que se pierde la posibilidad de que otras personas se puedan preparar, por lo que es necesario trabajar el abandono escolar desde la prevención (Martín, Alemán, Marchena y Santana, 2015). Asimismo, se debe enfocar, entre otros factores, en aquellos emanados de la familia como la participación parental en los estudios de sus hijas e hijos, compuesto por la protección, la exigencia, la supervisión, las aspiraciones hacia ellas y ellos, y la implicación con la propia escuela, así como el clima familiar, conformado por la armonía, la unidad y los valores (Gómez-Restrepo, Padilla y Rincón, 2016; Márquez, 2016; Beltrán y López, 2015; Villalba, 2013; El Sahili, 2011). Además, se agrega un factor psicológico del propio estudiantado, la confianza, construida por el estímulo al desempeño, el agrado a sí mismo, la identificación con la escuela y la seguridad emocional (Ricoy y Couto, 2018; Velázquez y González, 2017; Gómez y Vázquez, 2014).

\section{Contexto del estudio}

Aunque inició operaciones el 13 de febrero de 2017, es el 16 de enero de 2019 cuando se expide la Ley de Creación del Colegio de Bachilleres Militarizado General Mariano Escobedo del Estado de Nuevo León (CBM), un sistema que imparte EMS y capacitación para el trabajo en tres años; regido por un esquema de tiempo completo y bajo una modalidad militarizada. Este sistema fomenta, entre la población estudiantil, el amor a la patria y propicia su buen desarrollo ciudadano mediante la formación académica, física y el adiestramiento militar (Gobierno del Estado de Nuevo León, 2019). La misión es formar bachilleres líderes, capaces de desenvolverse como referentes de conocimiento, guías de la sociedad en el manejo económico, científico, formativo, cívico y ambiental en el ámbito local, nacional e internacional, con un alto concepto de honor, firme disciplina y trabajo de excelencia. Dentro de los objetivos se encuentra el aseguramiento de la calidad, al asegurar buenos números de eficiencia terminal, para lo que se requiere atender la deserción escolar (Colegio de Bachilleres Militarizado "General Mariano Escobedo" del Estado de Nuevo León, 2019).

Para el semestre de agosto-diciembre de 2018, el CBM contaba con una población de 2310 personas estudiantes distribuidas en cuatro planteles. Por ser un organismo de nueva creación, a la fecha, no contaba con egresadas o egresados, pero uno de los retos más importantes era la permanencia, pues 357 alumnas y alumnos habían desertado; esta cifra representa el $15.45 \%$.

A pesar de que el Colegio cuenta con programas de atención psicológica, orientación vocacional, apoyo académico mediante tutorías, maestras y maestros, guías y asesorías, actividades deportivas y artísticas para generar un sentido de pertenencia, y becas para apoyar económicamente a las familias y asegurar la permanencia educativa del estudiantado (Colegio de Bachilleres Militarizado "General Mariano Escobedo" del Estado de Nuevo León, 2019), todavía está lejos del 10.9\% de abandono escolar que se registra en el estado de Nuevo León, en México, para el ciclo escolar 2018-2019 en EMS (Secretaría de Educación del Estado de Nuevo León, 2019).

\section{MARCo TEÓRICO}

\section{Participación parental en los estudios de sus hijas e hijos y el clima familiar}

La participación de las madres y los padres es vital para abatir la deserción escolar. En efecto, más del $50 \%$ consideran que, si participan y se comprometen tanto en los estudios de sus hijas e hijos como con 
la institución a la que asisten, pueden evitar el abandono (Martínez, Rayón y Torrego, 2017; Velázquez y González, 2017; Secretaría de Educación Pública, 2014b).

Por consiguiente, no es de extrañar que los factores familiares sean de las principales causas que inciden en la permanencia o el abandono escolar (Martínez-Sánchez, Hernández-Arzola, Carrillo-Méndez, RomualdoPérez y Hernández-Miguel, 2013). Dichos factores comprenden la disfuncionalidad parental, la orientación, la motivación, los valores practicados en el hogar, la ayuda prestada de las madres y padres en tareas escolares de sus adolescentes así como las exigencias académicas, el compromiso, la unión familiar, las actitudes de la mamá y el papá hacia la academia, las expectativas sobre las hijas e hijos y el acercamiento y la participación en juntas escolares (Camacho, 2018; Corzo y Reyes, 2017; Instituto Nacional para la Evaluación de la Educación [INEE], 2017; Valdés y Sánchez, 2016).

También, se agrega el clima familiar, el seguimiento al desempeño, las actitudes protectoras, la elaboración de proyectos de vida en conjunto, los niveles de estudio de las madres y los padres, el conocimiento de las amistades de sus hijas e hijos, la composición parental, el número de hermanas y hermanos y de personas viviendo en un mismo hogar, las normas y la violencia doméstica, el afecto entre integrantes, el desarrollo de una cultura académica, la pobreza económica del hogar, la asistencia a cursos de formación para madres y padres, así como las actividades extraescolares de las hijas e hijos y la cooperación familiar en actividades sociales y del hogar (Gaxiola y González, 2019; Díaz y Osuna, 2017; Peña, Soto y Calderón, 2016; Antelm, Gil-López y Cacheiro-González, 2015; Consejo para la Evaluación de la Educación Tipo Medio Superior, 2012).

\section{Confianza del estudiantado}

Durante la adolescencia, el alumnado experimenta cambios biológicos y psicológicos propios de la etapa, los cuales pueden influir en su actuar educativo, especialmente en bachillerato (Secretaría de Educación Pública, 2008). Dentro de los principales factores asociados con la deserción escolar se encuentra, precisamente, la psicología del estudiantado y el ajuste, o desajuste, que esto genera en el clima escolar (Miranda, 2018; Corzo y Reyes, 2017).

Luego, se pueden incluir el grado de identificación con la institución educativa, la confianza y seguridad de la población estudiantil, la relevancia apreciada de los planes y programas, la creencia de que la escuela es un espacio de represión, la satisfacción con el ambiente académico, el interés, el esfuerzo, la estimulación y la motivación del propio alumnado hacia el estudio, los apoyos psicológicos que se reciben en la escuela, la autoestima, la autorresponsabilidad, el involucramiento en el estudio, la actitud y el comportamiento (Romero y Hernández, 2019; Ricoy y Couto, 2018; Cuéllar, 2017; Instituto Nacional para la Evaluación Educativa, 2017; Velázquez y González, 2017; Corona, Reyes, Martínez y Rivas, 2016);

También, algunas y algunos autores mencionan problemas de salud mental, la asertividad, el desequilibrio sensitivo, la pasividad, la personalidad, la afectividad, el manejo de emociones, la resiliencia, la empatía y la apatía (Martínez et al., 2017; Martín et al., 2015; Salvà-Mut et al., 2014).

\section{Procedimientos metodológicos}

El estudio es descriptivo y correlacional. Con base en el tamaño de la población, que fue de 2310, con un nivel de confianza de $95 \%$ y un margen de error de $5 \%$, se obtuvo un tamaño de la muestra de 330. Para medir el nivel de confianza y el margen de error, se utilizó la fórmula de población finita, la cual quedó de la siguiente manera: $\mathrm{n}=(\mathrm{NZ2} . \mathrm{pq}) / \mathrm{d} .(\mathrm{N}-1)+\mathrm{Z} 2 . \mathrm{pq}$

Donde:

$\mathrm{n}=$ tamaño de la muestra 
$\mathrm{N}=$ tamaño de la población

$\mathrm{Z}=$ valor de $\mathrm{Z}$ crítico, calculado en la tabla de área de la curva normal. Nivel de confianza

$\mathrm{d}=$ nivel de precisión absoluta

$\mathrm{p}=$ proporción aproximada del fenómeno de estudio en la población de referencia

$\mathrm{q}=$ proporción de la población de referencia que no presenta el fenómeno de estudio (1-p)

Se aplicó una encuesta, estandarizada por las personas autoras, al estudiantado activo distribuido en los cuatro planteles que se encontraban en operación académica, de primero, tercero y quinto semestre, que comprendieron 210 jóvenes varones y 140 mujeres, de acuerdo con la proporción por género del CBM. Se emplearon 20 encuestas más en caso de que alguna de ellas tuviera errores en su llenado. El muestreo empleado fue probabilístico con un tamaño del 17\%, con el que se logró una representatividad en los resultados. La medición fue por escala de Likert donde 1 significó Nada y 5 Mucho. La información se procesó en el software SPSS 22.

El cuestionario fue elaborado con base en la extensa revisión de la literatura, donde se propusieron variables para cada uno de los factores, o constructos (ver Tabla 1), así como la asociación que existe entre ellos. Los constructos fueron conformados mediante la prueba de fiabilidad Alfa de Cronbach ${ }^{\mathrm{a}}$ ) cercano o superior a 0.7, lo que se considera como aceptable (George y Mallery, 2003) para establecer que las variables miden un mismo constructo y están relacionadas (Welch y Comer, 1988). Para considerar que los resultados fueron estadísticamente significativos se estableció un .-valor menor a 0.05. En la Tabla 1 se proponen los factores o constructos y se describen sus respectivas variables marcadas en negritas.

El primer factor independiente, participación parental en los estudios de sus hijas e hijos, fue construido por seis variables: involucramiento de las madres y padres en los estudios, supervisión, aspiraciones, madres y padres protectores, exigencia e implicación en lo que sucede en la escuela. El resultado obtenidos mediante la prueba de fiabilidad a fue de 0.740 . Por lo tanto, para el presente trabajo se entiende como participación parental en los estudios de sus hijas e hijos la implicación, la vigilancia y los requerimientos que las madres y los padres ejercen en las actividades académicas de sus hijas e hijas, así como el grado de anhelo que tienen en sus logros educativos.

Para el segundo constructo independiente, clima familiar, los indicadores fueron: valores sólidos, armonía en el hogar y unidad familiar. La fiabilidad expresada fue prácticamente aceptable, con 0.689. Se define entonces el clima familiar como la cohesión entre los miembros de una familia, el ambiente estable y el sentido de interdependencia que percibe el estudiantado con respecto a las personas que conforman su hogar.

El tercer factor independiente, confianza del estudiantado, lo comprenden: seguridad emocional, estímulo al desempeño, agrado por sí mismo y misma, e identificación con la preparatoria. Se obtuvo un a de 0.721 , que conceptualiza la confianza del estudiantado como la determinación, motivación y autocomplacencia que tiene el alumnado y la compenetración existente con su preparatoria.

En cuanto al constructo dependiente, propensión de deserción escolar, las variables consideradas fueron: pensamiento de abandono y tener que abandonar. La fiabilidad obtenida fue de 0.696 , cercana a lo aceptable. Así, para la presente aportación, la propensión a la deserción escolar se da cuando el alumnado tiene ideas de suspender su EMS o debe desengancharse por motivos familiares o psicológicos. 
TABLA 1

Modelo teórico. Variables que conforman cada uno de los factores o constructos

\begin{tabular}{|c|c|c|}
\hline $\begin{array}{l}\text { Factor o } \\
\text { constructo }\end{array}$ & Variable & Pregunta \\
\hline \multirow{6}{*}{$\begin{array}{l}\text { Participación } \\
\text { parental en } \\
\text { los estudios } \\
\text { de sus hijas } \\
\text { e hijos }\end{array}$} & PP1 & $\begin{array}{l}\text { ¿En qué medida crees que tu familia se } \\
\text { involucra en tus estudios? }\end{array}$ \\
\hline & PP2 & $\begin{array}{l}\text { ¿Cuánta supervisión recibes de tus } \\
\text { padres? }\end{array}$ \\
\hline & PP3 & $\begin{array}{l}\text { ¿En qué nivel se encuentran las } \\
\text { aspiraciones de tus padres hacia ti } \\
\text { mientras estudias la preparatoria? }\end{array}$ \\
\hline & PP4 & ¿Qué tan protectores son tus padres? \\
\hline & PP5 & ¿Qué tan exigentes son tus padres? \\
\hline & PP6 & $\begin{array}{l}\text { ¿En qué medida están involucrados tus } \\
\text { padres con los que sucede en tu } \\
\text { escuela? }\end{array}$ \\
\hline \multirow{3}{*}{$\begin{array}{l}\text { Clima } \\
\text { familiar }\end{array}$} & $\mathrm{CF} 1$ & $\begin{array}{l}\text { ¿Qué tan sólidos son los valores } \\
\text { adquiridos en tu casa? }\end{array}$ \\
\hline & $\mathrm{CF} 2$ & $\begin{array}{l}\text { ¿Qué tan armónica es la relación } \\
\text { familiar en tu casa? }\end{array}$ \\
\hline & CF3 & ¿Qué tan unida es tu familia? \\
\hline \multirow{4}{*}{$\begin{array}{l}\text { Confianza } \\
\text { del } \\
\text { estudiantado }\end{array}$} & $C 21$ & $\begin{array}{l}\text { En general, ¿qué tan seguro o segura te } \\
\text { Sientes emocionalmente? } \\
\text { ¿Qué tanto te estimula desempeñarte }\end{array}$ \\
\hline & $\mathrm{CZ2}$ & $\begin{array}{l}\text { bien o dar el máximo en la } \\
\text { preparatoria? }\end{array}$ \\
\hline & $\mathrm{CZ3}$ & $\begin{array}{l}\text { ¿Qué tanto agrado sientes por ti misma } \\
\text { o mismo? }\end{array}$ \\
\hline & $\mathrm{CZ4}$ & $\begin{array}{l}\text { ¿Qué tan identificado te sientes con la } \\
\text { preparatoria? }\end{array}$ \\
\hline \multirow{2}{*}{$\begin{array}{l}\text { Propensión } \\
\text { de deserción } \\
\text { escolar }\end{array}$} & PDE1 & $\begin{array}{l}\text { ¿En qué medida has pensado } \\
\text { abandonar tus estudios de la } \\
\text { preparatoria? }\end{array}$ \\
\hline & PDE2 & $\begin{array}{l}\text { ¿En qué medida tienes que abandonar } \\
\text { tus estudios de preparatoria? }\end{array}$ \\
\hline
\end{tabular}

Fuente: Elaboración propia con información obtenida en la revisión teórica.

ANÁLisis De Resultados

\section{Niveles y correlaciones de participación parental en los estudios de sus hijas e hijos, clima familiar, confianza del estudiantado y propensión a la deserción escolar en el CBM}

Una vez obtenidos los promedios de las variables para conformar los constructos, se procedió con los resultados estadísticos descriptivos.

Como se aprecia en la Tabla 2, la media del constructo participación parental en los estudios de sus hijas e hijos fue de 3.87, es decir, las alumnas y los alumnos del CBM consideran que sus mamás y papás se involucran en sus estudios, ejercen supervisión a sus actividades escolares, tienen aspiraciones sobre ellas o ellos, son protectores y protectoras, exigentes, y se involucran en la escuela entre regular y suficiente. En cuanto a tener valores familiares sólidos, relaciones armónicas en el hogar y la unión parental, los resultados promediaron 4.11, entre suficiente y mucho. Para la confianza del estudiantado, las alumnas y los alumnos del CBM estiman que tienen seguridad emocional, dan el máximo en la preparatoria, tienen agrado por sí mismos y mismas, y se identifican con el CBM entre regular y suficiente con una media de 3.76. Finalmente, la propensión de deserción según el estudiantado del CBM es de entre poco y nada con 1.69. 
TABLA 2

Estadísticos descriptivos

\begin{tabular}{llll}
\hline Constructo & Media & Desviación Estándar & $\mathrm{N}$ \\
\hline $\begin{array}{l}\text { Participación parental en } \\
\text { los estudios de sus hijas e }\end{array}$ & 3.8766 & 0.80729 & 346 \\
hijos & 4.1125 & 0.79944 & 346 \\
$\begin{array}{l}\text { Clima familiar } \\
\begin{array}{l}\text { Confianza del } \\
\text { estudiantado }\end{array}\end{array}$ & 3.7632 & 0.80729 & 346 \\
$\begin{array}{l}\text { Propensión de deserción } \\
\text { escolar }\end{array}$ & 1.6986 & 0.93077 & 346 \\
\hline
\end{tabular}

Fuente: Elaboración propia con información obtenida en trabajo de campo.

TABLA 3

Correlaciones

\begin{tabular}{lllll}
\hline Constructo & $\begin{array}{l}\text { Participación } \\
\text { parental en los } \\
\text { estudios de sus } \\
\text { hijas e hijos }\end{array}$ & $\begin{array}{l}\text { Clima } \\
\text { familiar }\end{array}$ & $\begin{array}{l}\text { Confianza del } \\
\text { estudiantado }\end{array}$ & $\begin{array}{l}\text { Propensión de } \\
\text { deserción } \\
\text { escolar }\end{array}$ \\
\hline $\begin{array}{l}\text { Participación } \\
\text { parental en los } \\
\text { estudios de sus } \\
\text { hijas e hijos }\end{array}$ & - & $0.434^{* *}$ & $0.212^{* *}$ & $-0.058^{* *}$ \\
$\begin{array}{l}\text { Clima familiar } \\
\begin{array}{l}\text { Confianza del } \\
\text { estudiantado }\end{array}\end{array}$ & - & $0.366^{*}$ & $-0.102^{* *}$ \\
$\begin{array}{l}\text { Propensión de } \\
\text { deserción escolar }\end{array}$ & & - & $-0.25^{*}$ \\
\hline
\end{tabular}

Fuente: Elaboración propia con información obtenida en el trabajo de campo.

Los resultados fueron estadísticamente significativos a un nivel de confianza de ${ }^{*} \mathrm{p}<, 05 ;{ }^{* *} \mathrm{p}<, 01 ;{ }^{* * *} \mathrm{p}<, 001$

En seguida, se obtuvieron las correlaciones entre factores (Tabla 3), donde se resaltan las que hay entre los constructos independientes, así como las correlaciones negativas que el factor dependiente tiene con todas las independientes.

\section{Participación parental en los estudios de sus hijas e hijos, clima familiar, confianza del estudiantado y su determinación hacia la propensión a la deserción escolar en el CBM}

Para comprobar las relaciones entre los constructos expuestos en el modelo teórico, se aplicó una técnica de regresión lineal múltiple representada de la siguiente forma:

$$
y=\beta 0+\beta 1 \mathrm{X} 1+\beta 2 \mathrm{X} 2+\beta 3 \mathrm{X} 3+\mathrm{e}
$$

Donde:

$\mathrm{X} 1=$ Participación parental en los estudios de sus hijas e hijos

$\mathrm{X} 2$ = Clima familiar

$\mathrm{X} 3$ = Confianza del estudiantado

$\mathrm{y}=$ Propensión de deserción escolar en el CBM 
En la Tabla 4 se aprecia que el modelo tiene un nivel explicativo significativo del $5.4 \%$ de la varianza $(\mathrm{R} 2=$ 0.054), que con un $\mathrm{p}$ - valor < .001 representa el grado de explicación del factor dependiente con respecto a los independientes, o bien, la relación que hay entre las . con la .. Con el valor arrojado, y con base en los muchos y complejos factores que intervienen en la deserción escolar, se puede suponer que el modelo es confiable. Se entiende que la propensión de deserción escolar entre el alumnado activo del CBM aumentará o disminuirá según el clima familiar y la confianza del propio estudiantado, mientras que la $\beta$ que arroja el modelo empírico para el factor participación parental es de 0 , por lo que indica que no influye.

La Figura 1 refleja las asociaciones que hay de manera directa: de forma negativa, la confianza y el clima familiar tienen un peso de -0.246 y - 0.014 , respectivamente; mientras que la participación de los padres y las madres en los estudios de sus hijas e hijos registró un peso de 0.00 , por lo que se establece:

$$
\mathrm{y}=2.685-0.014 \times 2-0.246 \times 3
$$

[Ecuación 2]

TABLA 4

Estadísticas de regresión

\begin{tabular}{ll}
\hline Estadísticas de la regresión & \\
\hline P - Valor & $0.001^{* * *}$ \\
Coeficiente de correlación múltiple & 0.250 \\
Coeficiente de determinación $\mathrm{R}^{\wedge} 2$ & 0.054 \\
Error tipico & 0.9052 \\
Durbin-Watson & 1.893 \\
\hline
\end{tabular}

Fuente: Elaboración propia con información obtenida en el trabajo de campo.

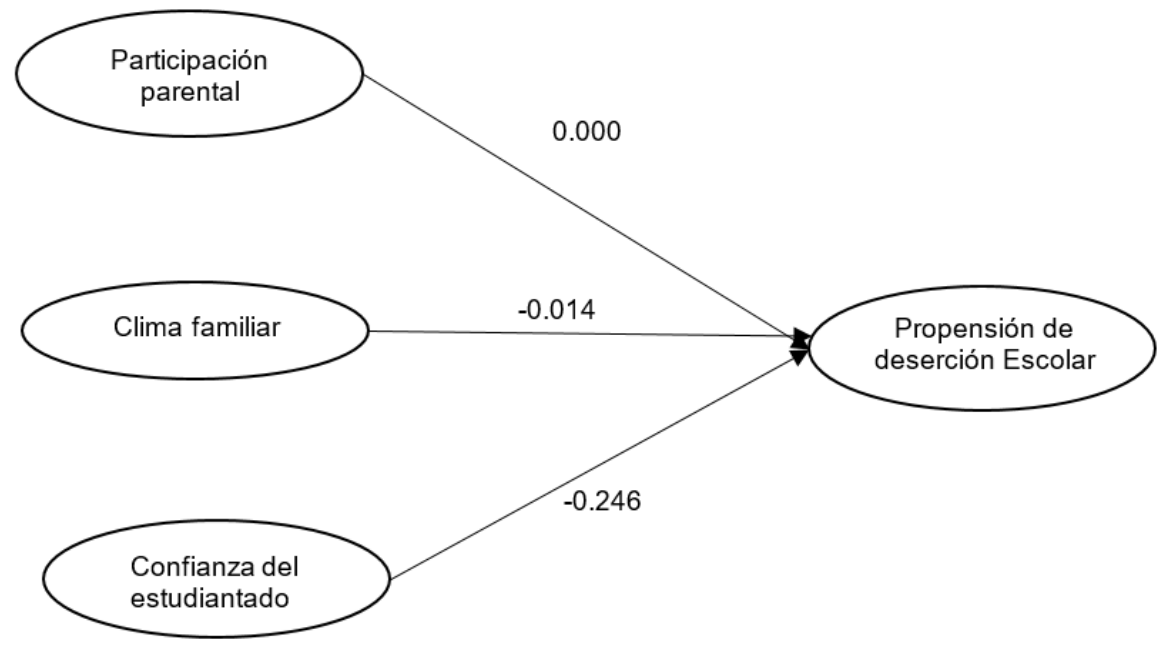

FIGURA 1.

Modelo empírico

Fuente: Elaboración propia con información obtenida por el trabajo de campo.

\section{Discusión de resultados}

a participación parental en las actividades académicas de sus hijas e hijos no se asocia con la propensión de deserción escolar del alumnado del CBM (Figura 1). Con respecto a lo anterior, Miranda (2018) afirma que los motivos principales del abandono son económicos, individuales y escolares, sobre todo aquellos relacionados con la calidad educativa, capacitación del profesorado y la infraestructura y equipamiento; 
Cuéllar (2017) y Ruíz-Ramírez et al. (2014) agregaron que la mayoría de los abandonos se dan de manera voluntaria, estos se centran en aspectos sociales y acciones académicas del propio estudiantado, como la inasistencia y reprobación; Corona et al. (2016) mencionaron la deficiencia en el conocimiento con el que llegan al bachillerato, así como la falta de adaptación de las personas docentes a las nuevas exigencias. Barrales, Gómez y Guerrero (2015) consideraron el actuar docente en clases y añadieron la capacidad cognitiva del alumnado y los hábitos de estudio.

Además, las investigaciones de Román (2013) dieron más peso a la condición socioeconómica, mientras que De la Peña (2014) ubicó que el 70\% de las personas desertoras lo hicieron por motivos personales y económicos, aunque señaló la comunicación de madres y padres con sus hijas e hijos como variable de protección. López y Beltrán (2017) informaron sobre desacertada elección de la escuela que no empata con las necesidades y deseos del alumnado.

En seguida, el clima familiar se asoció negativamente con la propensión de deserción escolar por parte del alumnado del CBM. Aunque no es considerable, sin duda los valores adquiridos en el hogar, la armonía y la unión, son positivos para el estudiantado adolecente, y esto influye en la convicción propia para continuar sus estudios de bachillerato. Lo anterior concuerda con lo que informan Gómez-Restrepo et al. (2016), quienes identificaron ambientes ríspidos en la familia como una de las principales causas de reprobación y posterior abandono. Camacho (2018) y Romero y Hernández (2019) agregaron la descomposición familiar y la deficiencia de valores familiares como causas de peso para desertar los estudios, pues se replican y ponen en práctica en los centros educativos. Los resultados de Vera (2012) refuerzan la asociación que hay entre el abandono con el clima, la composición y los valores familiares.

Finalmente, la confianza del estudiantado también se asocia negativamente con pretender desertar de la escuela. Los resultados se alinean con lo publicado por la CEJC (2013), donde se concluyó que la desmotivación es el primer factor de deserción escolar con un 65.2\%; se refuerza con lo manifestado por Díaz y Osuna (2017), quienes identificaron la desmotivación y el desagrado por el estudio como unos de los factores principales de abandono. Velasco y Moreno (2016) ubicaron el valor que el propio alumnado le da al bachillerato; mientras que Ruiz-Ramírez et al. (2014) apuntaron la automotivación. El INEE (2017) informó que robustecer la identidad de las personas adolescentes con la institución neutraliza la deserción; así como elevar la intensidad de su propia seguridad emocional (Márquez, 2016).

\section{Conclusiones y recomendaciones}

Los objetivos de la investigación se cumplieron. Se asoció la participación parental en los estudios de sus hijas e hijos, el clima familiar y la confianza del estudiantado, con la propensión de deserción escolar de las alumnas y los alumnos activos del CBM; además, se obtuvieron los niveles de dichos factores entre la población estudiantil del CBM.

La tendencia central es que el alumnado del CBM considera que la participación de sus madres y padres en sus estudios se da de manera regular, muy cercana a suficiente, es decir, se involucran en sus estudios, ejercen supervisión, tienen suficientes aspiraciones académicas para sus hijas e hijos, son protectoras y protectores, exigentes ante los desafíos escolares y se involucran con lo que sucede en el colegio. La percepción de su clima familiar se ubicó entre suficiente y mucho, lo que significa que la alumna o el alumno percibe sólidos valores, armonía y unidad en el seno familiar. La confianza que el estudiantado tiene sobre sí se ubicó entre regular y suficiente, es decir, el alumnado se siente seguro emocionalmente, lo que estimula el desempeño óptimo, pues siente agrado por sí mismo y se identifica con el CBM. Finalmente, la población estudiantil del CBM ha pensado o ha tenido que desertar en un nivel superior a nunca pero menos que poco.

Por último, el factor dependiente está determinado por los factores independientes en solo 0.054; sin embargo, por la complejidad del fenómeno del abandono escolar en la EMS, es conveniente señalarlo. La participación parental en los estudios de sus hijas e hijos no influye en la propensión de deserción escolar en 
el CBM, mientras que el clima familiar impacta negativamente en un $1.4 \%$ y la confianza del estudiantado lo hace, igualmente de forma negativa, en un $24.6 \%$.

Por lo tanto, se recomienda, más allá de que el involucramiento de las madres y los padres con los estudios de sus hijas e hijos que no se asoció con la pretensión de desertar del CBM, fomentar la participación parental en el desempeño académico del estudiantado, así como el involucramiento de los alumnos y alumnas en actividades escolares como desfiles, celebraciones y acciones comunitarias; asimismo, que los actos de exigencia, supervisión, protección y aspiración hacia sus hijas e hijos no sean excesivos pero tampoco relajados. El equilibrio no sofoca al estudiantado, pero permite un control efectivo del desempeño, así como su correcto desarrollo educativo. El constante estudio y deseos de superación de los padres y las madres de familia también son favorables, así como evitar las adicciones y las influencias negativas entre adolescentes.

Para mejorar el clima familiar se recomienda propiciar espacios familiares de estimulación para la lectura, reflexión y aprendizaje; concientizar sobre el valor real de las cosas inmateriales, como el estudio, el conocimiento y la superación integral; el uso de redes sociales para como medio de información acerca de actividades escolares y del propio estudiantado; fomentar la cooperación familiar y las tareas compartidas que unan a los integrantes tenderán a arrojar resultados favorables contra la deserción escolar en el CBM.

Luego, para reforzar la confianza del estudiantado, se propone integrar al alumnado en actividades académicas, deportivas, culturales y artísticas organizadas por el CBM, esto para acrecentar su sentido de pertenencia con la institución. Los apoyos psicopedagógicos identificarán las debilidades de la población estudiantil, para así proponer estrategias con miras a fortalecer su autoestima, seguridad emocional y su motivación por el estudio; promover ambientes académicos seguros e incluyentes, que den tranquilidad y satisfacción mejoran la permanencia: los trabajos en equipos suelen ser una buena opción, así como estimular el aprendizaje autónomo, programar coloquios sobre proyectos de vida, planes de carrera, manejo de emociones y valores, principalmente durante los cursos inductivos o el primer ciclo escolar. También, crear espacios comunes para que madres, padres, estudiantes y docentes convivan con miras a medir y estimular el avance del alumnado y su permanencia en el CBM.

Por último, sería conveniente realizar más estudios que establezcan si los factores familiares o psicológicos aquí abordados favorecen a la motivación o la solidaridad entre integrantes de las familias, y si a su vez inciden en la permanencia del estudiantado en el CBM, o bien, cómo otros factores — pedagógicos o escolaresinciden en la tendencia de deserción escolar en el CBM.

\section{ReFERENCIAS BIBLIOgRÁFICAS}

Antelm, A., Gil-López, A. y Cacheiro-González, M. (2015). Análisis del fracaso escolar desde la perspectiva del alumnado y su relación con el estilo de aprendizaje. Revista Educación y Educadores, 18(3), (pp. 471-489). doi: https://doi.org/10.5294/edu.2015.18.3.6

Barrales, A., Gómez, A. y Guerrero, L. (2015). Factores que originan la reprobación en los estudiantes de bachillerato: caso Colegio Motolinia. En: J.A. Hernanz y M.L. Watty (Coords.), Tendencias y desafios en la innovación educativa: un debate abierto. Veracruz, México: Fundación para la Educación Superior Internacional, AC. (pp. 881-890). Recuperado de https://www.uv.mx/dgdaie/files/2016/10/E-book-TDIE.pdf

Beltrán, L. y López, L. (2015). Causas de abandono escolar según padres de familia del nivel superior. Pistas Educativas, 35(111), (pp. 5-19). Recuperado de http://www.itcelaya.edu.mx/ojs/index.php/pistas/article/view/353

Camacho, M. (2018). Fracaso escolar y abandono educativo temprano en Huelva. Prácticas en Positivo y propuestas de mejora. España: Universidad Internacional de Andalucía.

Colegio de Bachilleres Militarizado “General Mariano Escobedo" del Estado de Nuevo León (2019). Plan de Desarrollo Institucional 2019-2024. México. 
Consejería de Educación de la Junta de Castilla y León [CEJC]. (2013). Abandono escolar temprano. Factores de abandono y factores de continuidad. Intervenciones posibles desde los centros educativos. España. Recuperado de ht tp://www.itcelaya.edu.mx/ojs/index.php/pistas/article/view/353

Consejo para la Evaluación de la Educación del Tipo Medio Superior (2012). Reporte de la Encuesta Nacional de Deserción en la Educación Media Superior. México: Secretaría de Educación Pública.

Corona, V., Reyes, S., Martínez, S. y Rivas, C. (2016). Estrategias para la disminución de los índices de reprobación en el Instituto Tecnológico de Pachuca. Revista de sistemas y Gestión Educativa, 3(9), (pp. 62-69). Recuperado de https://bit.ly/3aNAv05

Corzo, C. y Reyes, C. (2017). Principales causas de reprobación de alumnos de los grupos de quinto semestre, grupo seis y ocho de la escuela preparatoria número tres. Con-Ciencia, 4(7). doi: http://doi.org/10.4000/configuraco es.2309

Cuéllar, D. (2017). Abandono escolar en educación media superior. Análisis de factores escolares asociados a altas tasas de abandono. México: Congreso Nacional de Investigación Educativa.

De la Peña, G. (2014). El problema de la deserción escolar y las posibilidades de la investigación-acción participativa. Configuraciones. Revista de Sociología 14, 1-18. doi http://doi.org/10.4000/configuracoes.2309

Díaz, K. y Osuna, C. (2017). Contexto sociofamiliar en jóvenes en situación de abandono escolar en educación media superior. Perfles Educativos, 39(158), 70-91. Recuperado de http://www.scielo.org.mx/pdf/peredu/v39n158/ 0185-2698-peredu-39-158-00070.pdf

El Sahili, L. (2011). Informe de investigación sobre deserción escolar mediante jerarquización de factores en la UPIIG del IPN en Silao, Guanajuato. REXE. Revista de Estudios y Experiencias en Educación, 10(19), 33-48. Recuperado de https://www.redalyc.org/articulo.oa?id=243120126002

Gaxiola, J.y González, S. (2019). Apoyo percibido, resiliencia, metas y aprendizaje autorregulado en bachilleres. Revista Electrónica de Investigación Educativa, 21, 1-10. doi: http://doi.org/10.24320/redie.2019.21.e08.1983

George, D. y Mallery, P. (2003). SPSS for Windows step y step: A simple guide and reference. (4a Ed.). Boston: Pearson.

Gobierno del Estado de Nuevo León (2019). Ley que Crea al Colegio de Bachilleres Militarizado "General Mariano Escobedo" del Estado de Nuevo León, (pp. 17-37). Nuevo León, México: Gobierno del Estado de Nuevo León. Recuperado de https://normas.cndh.org.mx/Documentos/Nuevo\%20Le\%C3\%B3n/Ley_CCBMGMEE_NL .pdf

Gómez, M. y Vázquez. E. (2014). Deserción a nivel medio superior en escuela superior Tepeji. Tepexi. Boletín Cientifico de la Escuela Superior Tepeji, 1(2), 1-5.

Gómez-Restrepo, C., Padilla, A. y Rincón, C. (2016). Deserción escolar de adolescentes a partir de un estudio de corte transversal: Encuesta de Salud Mental Colombia 2015. Revista Colombiana de Psiquiatría, 45(1), 105-112. doi: https://doi.org/10.1016/j.rcp.2016.09.003

Instituto Nacional para la Evaluación de la Educación [INEE]. (2017).Directrices para mejorar la permanencia escolar en la educación media superior. Recuperado de https://www.inee.edu.mx/wp-content/uploads/2018/12/P1F1 05.pdf

Landeros, J. (2012). Deserción en la educación media superior en México (Tesis de Licenciatura). Instituto tecnológico y de estudios superiores de Monterrey, México. Recuperado de https://repositorio.tec.mx/bitstream/handle/1 1285/631813/33068001103948.pdf?sequence $=1$

López, L. y Beltrán A. (2017). La deserción en los estudiantes de educación superior: tres percepciones en estudio, alumnos, docentes y padres de familia. Pistas Educativas, 39(126), 143-159.

Márquez, C. (2016). Factores asociados al fracaso escolar en la educación secundaria de Huelva. REICE Revista Iberoamericana sobre Calidad, Eficacia y Cambio en Educación, 14(3), 131-144. doi: https://doi.org/10.15366 /reice2016.14.3.007

Martín, J., Alemán, J., Marchena, R. y Santana, R. (2015). El contexto familiar del alumnado en riesgo de abandono escolar temprano según la tipología familiar. Profesorado. Revista de Currículum y Formación del Profesorado, 19(3), 246-263. 
Martínez, M., Rayón, L. y Torrego, J. (2017). Las familias ante el abandono escolar. Bordón. Revista de Pedagogía, 69(2), 59-70. Recuperado de: https://dialnet.unirioja.es/servlet/articulo?codigo=5901375

Martínez-Sánchez, A., Hernández-Arzola, L., Carrillo-Méndez, D., Romualdo-Pérez, Z. y Hernández-Miguel, C. (2013). Factores asociados a la reprobación estudiantil en la Universidad de la Sierra Sur, Oaxaca. Temas de Ciencia y Tecnología, 17(51), 25-33.

Miranda, F. (2018). Abandono escolar en educación media superior: conocimiento y aportaciones de política pública. Sinética. Revista Electrónica de Educación, 51, 1-22. doi: http://doi.org/10.31391/s2007-7033(2018)0051-010

Peña, J., Soto, V. y Calderón, U. (2016). La influencia de la familia en la deserción escolar. Revista Mexicana de Investigación Educativa, 21(70), (pp. 881-899).

Poy, R. (2010). Efectos del credencialismo y las expectativas sociales sobre el abandono escolar. Revista de Educación, número extraordinario, 147-169.

Ricoy, M. y Cauto, M. (2018). Desmotivación del alumnado de secundaria en la materia de matemáticas. Revista Electrónica de Investigación Educativa, 20(3), 69-79. doi: http://doi.org/10.24320/redie.2018.20.3.1650

Román M. (2013). Factores asociados al abandono y la deserción escolar en América Latina: Una mirada en conjunto. REICE. Revista Iberoamericana sobre Calidad, Eficacia y Cambio en la Educación, 11(2), 33-59. Recuperado de https://www.redalyc.org/pdf/551/55127024002.pdf

Romero, E. y Hernández, M. (2019). Análisis de las causas endógenas y exógenas del abandono escolar temprano: una investigación cualitativa. Educación XX1, 22(1), 263-293. doi: https://doi.org/10.5944/educXX1.21351

Ruiz-Ramírez, R., García-Cue, J. y Pérez-Olvera, M. (2014). Causas y consecuencias de la deserción escolar en el bachillerato: Caso Universidad Autónoma de Sinaloa. Ra Ximhai, 10(5), 51-74.

Salvà-Mut, F., Oliver-Trobat, M. y Comas-Forgas, R. (2014). Abandono escolar y desvinculación de la escuela: perspectiva del alumnado. Revista Internacional de Investigación en Educación, 6(13), 129-142. Recuperado de h ttps://www.redalyc.org/pdf/2810/281031320009.pdf

Secretaría de Educación del Estado de Nuevo León (2019). Indicadores Educativos. México. Recuperado de https://w ww.nl.gob.mx/publicaciones/alumnos-por-grado-municipio-y-sostenimiento

Secretaría de Educación Pública (2008). Acuerdo número 442 por el que se establece el Sistema Nacional de Bachillerato en un marco de diversidad. México: SEP. Recuperado de https://bit.ly/3j9oHYs

Secretaría de Educación Pública (2014a). Caja de herramientas para la gestión contra el abandono escolar. México: SEP. Recuperado de https://bit.ly/2WpN4rn

Secretaría de Educación Pública (2014b). Yo no abandono 2. Manual para recibir a los nuevos estudiantes en planteles de educación media superior: México: SEP.

Secretaría de Educación Pública (2014c). Yo no abandono 11. Manual para el desarrollo de habilidades socioemocionales en planteles de educación media superior: México: SEP.

Silvera, L. (2016). La evaluación y su incidencia en la deserción escolar: ¿falla de un sistema, de las instituciones educativas, del docente o del estudiante? Revista Educación y Humanismo, 18(31), 13-325.

Velasco, A. y Moreno G. (2016). Abandono escolar en una institución de educación media superior en Jiutepec. Inventio, la génesis de la cultura universitaria en Morelos, 12(27), 1-9. Recuperado de: http://inventio.uaem.mx /index.php/inventio/article/view/132/419

Valdés, A. y Sánchez, P. (2016). Las creencias de los docentes acerca de la participación familiar en la educación. Revista Electrónica de Investigación Educativa, 18(2), 105-115. Recuperado de http://www.scielo.org.mx/pdf/redie/v1 8n2/1607-4041-redie-18-02-00105.pdf

Velázquez, Y. y González, M. (2017). Factores asociados a la permanencia de estudiantes universitarios: Caso UAMMUAT. Revista de Educación Superior, 46(184), 117-138. doi: http://doi.org/10.1016/j.resu.2017.11.003

Vera, L. (2012). Características de familias de adolescentes desertores escolares matriculados en capacitación laboral. MedULA Revista de Facultad de Medicina. 21(1). 47-51. Recuperado de https://bit.ly/3xwk2Y4 
Vidales, S. (2009). El fracaso escolar en la educación media superior: El caso del bachillerato de la Universidad Mexicana. Revista Iberoamericana sobre Calidad, Eficacia y Cambio en Educación, 7(4), 320-341. Recuperado de https://revistas.uam.es/index.php/reice/article/view/5395

Villalba, T. (2013). ¿Cómo influye la estructura familiar en la probabilidad de fracaso escolar de los adolescentes en España? En H. Cairo y L. Finkel (Coords). Crisis y cambio: propuestas desde la sociología, Actas del XI Congreso Español de Sociología: Vol. II. XI Congreso Español de Sociología. (pp. 230-240).Universidad Complutense de Madrid (UCM), Madrid, España. Recuperado de https://dialnet.unirioja.es/servlet/articulo?codigo=7285994

Welch, S. y Comer, J. (1988). Quantitative Methods for Public Administration: Techniques and Applications. Estados Unidos: Waveland Pr Inc.

\section{INFORMACIÓN ADICIONAL}

Cómo citar: Lozano-Treviño, D.F. y Maldonado-Maldonado, L. (2021). Asociación entre participación parental en los estudios, clima familiar y confianza del estudiantado con la propensión de deserción escolar del Colegio de Bachilleres Militarizado "General Mariano Escobedo" del Estado de Nuevo León (CBM). Revista Educación, 45(2). Recuperado de http://doi.org/10.15517/revedu.v45i1.42846 